\title{
Predictores psicológicos de la re-ganancia del peso en pacientes bariátricos
}

\author{
Psychological predictors of the regain of weight in bariatric patients
}

\author{
Carla Ugarte \\ Álvaro Quiñones \\ Universidad San Sebastián, Chile \\ Benjamín Vicente \\ Universidad de Concepción, Chile \\ Recibido (25 de marzo de 2019) Aceptado (09 de mayo de 2019)
}

\begin{abstract}
Resumen
La cirugía bariátrica permite una pérdida ponderal significativa y acelerada en el corto plazo. La re-ganancia del peso se observa entre el 15 al 30\% de los pacientes. Los factores psicológicos explicarían en mayor medida la recuperación del peso en pacientes bariátricos. El objetivo del presente estudio fue evaluar la capacidad predictiva de las variables "locus de control del peso", "perspectiva temporal" y "autoeficacia, para la re-ganancia del peso en pacientes bariátricos. Estudio multivariado predictivo. Se evalúo a 97 pacientes bariátricos. Los resultados indican que las variables psicológicas "locus de control", "presente fatalista" y "pasado positivo" explican el $27.1 \%$ de la varianza de la re-ganancia del peso ( $p<.001)$. Este modelo clasifica de forma correcta a un $88.7 \%$ de los pacientes. Las variables locus de control del peso, pasado positivo y presente fatalista predicen la re-ganancia del peso.

Palabras clave: Cirugía bariátrica, locus de control, autoeficacia, perspectiva temporal, predictores de la re-ganancia.
\end{abstract}

\begin{abstract}
Bariatric surgery allows a significant and accelerated weight loss in the short term. The regain of the weight is observed between 15 to $30 \%$ of the patients. The psychological factors would explain to a greater extent the recovery of weight in bariatric patients. Aim: To evaluate the predictive capacity of the variables "locus of weight control", "temporal perspective" and "self-efficacy", for the re-gain of weight in bariatric patients. Method: Predictive multivariate study. A total of 97 bariatric patients were evaluated. Results: The psychological variables "locus of control", "present fatalist" and "positive past" explain $27.1 \%$ of the variance of the regain of weight $(\mathrm{p}<.001)$. This model correctly classifies $88.7 \%$ of patients. Conclusion: The variables locus of weight control, positive past and fatalistic present predict the regain of weight.

Key Words: Bariatric Surgery, Locus of Control, Self-efficacy, Time Perspective, Predictors of regain.
\end{abstract}

Correspondencia: Dr. Benjamín Vicente. Maipú No 2120, Concepción. Correo: bvicent@udec.cl Teléfono: 56-41-2204299. Agradecimientos: A los pacientes que participaron voluntariamente del presente estudio. Especialmente le agradezco al Dr. Gerardo Astete (Q.E.P.D) por su ayuda para la realización de la presente investigación, y a los psicólogos Fernanda Mena y Jorge Figueroa que en ese momento eran parte del Equipo de Tratamiento Quirúrgico para la Obesidad de Concepción (ETO-Conce). 


\section{Introducción}

La cirugía bariátrica actualmente es el tratamiento más eficaz para tratar la obesidad (Adams et al., 2017; Schauer et al., 2017). Al permitir una pérdida del peso de forma acelerada y por mayor tiempo (Courcoulas et al., 2018; Adams et al., 2017; Maciejewski et al., 2016; Adams et al., 2012). No obstante, si el paciente no acompaña este proceso con cambios en el estilo de vida a largo plazo facilitará recidivas (Jiménez et al., 2018). De hecho entre un 20 a 30\% de los pacientes que se someten a cirugía bariátrica no pierden el porcentaje de exceso de peso esperado y/o recuperan un porcentaje significativo del de peso perdido en el mediano plazo (Clapp, Wynn, Martyn, Foster, O’Dell \& Tyroch, 2018; Jiménez et al., 2018; Amundsen, Strommen \& Martins, 2017; Lemanu, Singh, Rahman, Hill, Babor \& MacCormick, 2015).

Diversos equipos de investigadores (Van de Laar, van Rijswijk, Kakar \& Bruin, 2018; Sabench, et al., 2017; Corcelles, Daigle, \& Schauer, 2016; van de Laar, 2012), han recomendado expresar los resultados de pérdida de peso utilizando el "Porcentaje de Peso Total Perdido" (\%PTP) o \%TWL (Percentage Total Weight Loss). El $\%$ PTP se considera exitoso cuando es igual o superior al 25\% (van de Laar et al. 2018). Por otra parte, la re-ganancia del peso suele iniciarse próxima al alta del paciente por parte del equipo multidisciplinario (Lemanu et al., 2015; Himpens, Dobbeleir \& Peeters, 2010), y se define como la recuperación del 15\% o más de la pérdida del peso desde el nadir (menor peso registrado post-cirugía) (Shukla, He, Saunders, Andrew \& Aronne, 2018; Cooper, Simmons, Webb, Burns \& Kushner, 2015; Odom et al., 2010). En relación al peso y su evolución en pacientes bariátricos, la investigación muestra que los datos antropométricos autorreportados son confiables (Ivezaj \& Grilo, 2017; Jenkins et al., 2016).

En la actualidad, existe diversa evidencia acerca que la re-ganancia del peso se debe principalmente a la no adherencia a pautas alimentarias saludables y de actividad física, asociadas a variables psicológicas (Busetto et al., 2017; Kushner \& Sorensen, 2015; Anastasiou et al., 2015; Neymotin \& Nemzer, 2014).

En este contexto, diversas variables psicopatológicas han sido estudiadas en relación a la re-ganancia del peso en pacientes bariátricos (Wedin et al., 2014; Legenbauer, Petrak, de Zwaan \& Herpertz, 2011), con resultados inconsistentes (Alfonsson, Weineland-Strandskov \& Sundbom, 2017; Dawes et al., 2016; Wedin et al., 2014; Beck, Mehlsen \& Stoving, 2012; Legenbauer, et al., 2011; Franks \& Kaiser, 2008; Herpetz, Kielmann \& Wolf, 2004). Por lo anterior, desde hace algunos años, se han estudiado en este campo variables psicológicas de tipo cognitivo-actitudinal como autoeficacia, locus de control y perspectiva temporal (Peterhänsel, Linde, Wagner, Dietrich \& Kersting, 2017; Nickel, Schmidt, Bruckner, Büchler, Müller-Stich \& Fischer, 2017; Lent et al., 2016; Anastasiou, Fappa, Karfopoulou, Gkza, \& Yannakoulia, 2015; Neymotin \& Nemzer, 2014; González \& Lugli, 2012; Hall, Fong \& Cheng, 2012).

La "Autoeficacia para el control del peso" se refiere a las creencias acerca de las capacidades personales para organizar y ejecutar los cursos de acción necesarios para controlar el peso corporal (González \& Lugli, 2012). Una alta autoeficacia ha sido asociada a la intención de realizar conductas saludables y a la pérdida del peso al inicio de los tratamientos (Menéndez-González \& Orts-Cortés, 2018; Campos, Argüelles, Vázquez, \& Ortiz, 2014; Latner, McLeod, O’Brien \& Johnston, 2013; Bas \& Donmez, 2009; Campos \& Pérez, 2007).

El "Locus de control" hace referencia a las creencias personales acerca del grado de control que se tiene sobre los acontecimientos vitales (Rotter 1966). Se diferencian dos tipos de locus de control. 1) Locus de Control Interno (LCI): creencia de que los acontecimiento son contingentes a la conducta de la persona; 2) Locus de Control Externo (LCE): creencia de que los acontecimientos no dependen del comportamiento, sino que de la suerte, la casualidad, el destino o el poder de otros. Por lo anterior, las personas que presentan un locus de control interno asumen mayor responsabilidad sobre los acontecimientos vitales (Rotter, 1966).

De igual manera, el locus de control tiene efectos sobre el estado psicológico y la calidad de vida de pacientes con distintas condiciones y/o enfermedades crónicas como la obesidad (Menéndez-González \& Orts-Cortés, 2018; Anastasiou, et al., 2015; Neymotin \& Nemzer, 2014). La investigación evidencia una relación positiva entre el locus de control interno y completar un programa de pérdida de peso de forma exitosa (Balch \& Ross, 1975). Además, el locus de control se ha asociado a la mantención de la pérdida en pacientes con sobrepeso u obesidad (Neymotin \& Nemzer, 2014; Morowati, Mazlomy, Baghiani \& Rouhani, 2009).

La perspectiva temporal se define como "el proceso no consciente mediante el cual los flujos continuos de experiencias personales y sociales se asignan a categorías temporales, o marcos temporales, que ayudan a dar orden, coherencia y significado a esos eventos" (Zimbardo \& Boyd, 1999, p.1271). Las personas tienen una 
focalización relativamente estable en una de las tres dimensiones temporales: pasado, presente, futuro; con consecuencias en sus cogniciones, emociones y conductas (Zimbardo \& Boyd, 2009).

La perspectiva temporal puede dividirse en cinco dimensiones: Pasado Positivo (PP), Pasado Negativo (PN), Presente Hedonista (PH), Presente Fatalista (PF) y Futuro (F) (Zimbardo \& Boyd, 1999). Por ejemplo, personas con perspectiva de futuro predominante se guían por objetivos, el logro de metas y la planificación. También, poseen un perfil de posponer la recompensa e informan altos niveles de conductas de salud. Por el contrario, las personas con una perspectiva hedonista están mayormente centradas en la búsqueda del disfrute, focalizados en consecuencias y eventos inmediatos (Daugherty \& Brase, 2010; Wills, Sandy \& Yaeger, 2001). Por otra parte, las personas con una perspectiva de tiempo fatalista se centran una actitud negativa hacia las vivencias y desesperanza, por lo que tienden a ignoran en gran medida las consecuencias de su comportamiento y presentan conductas más irresponsables con prácticas destructivas en salud (Keough, Zimbardo \& Boyd, 1999; Henson, Carey \& Carey, 2006).

Cuando una dimensión de la perspectiva temporal domina, minimiza o excluye las otras se vuelve disfuncional (Boyd \& Zimbardo, 2005). Por ejemplo, una predominancia única en el Pasado Positivo presenta inconvenientes que pueden incluir ser excesivamente conservador, cauteloso, con miedo al cambio y la apertura a nuevas experiencias y culturas, deseo de mantener el status quo y aplicar soluciones antiguas a nuevos problemas.

Específicamente, en la esfera de la salud mental, la naturaleza temporal del comportamiento humano tiene relevancia explicativa en diferentes áreas como por ejemplo: trastornos psicopatológicos (Ryu, Kook, Lee, Ha \& Cho, 2014), emociones y desórdenes del ánimo (Oyanadel \& Buela-Casal, 2017; Droit-Volet, 2013), Psicoterapia (Quiñones, Ceric, Ugarte \& Pascale, 2017; Quiñones, Ceric \& Ugarte, 2015); adherencia en pacientes diabéticos (Baird, Webb, Martin \& Sirois, 2018; Quiñones, Ugarte, Chávez \& Mañalich, 2018; Hall, Fong \& Cheng, 2012); y, recuperación del peso en pacientes bariátricos (Alfonsson et al., 2017; Ugarte, Quiñones \& Vicente, 2019 en prensa; Ugarte, Quiñones, Bustos \& Vicente, 2020 en prensa).

En relación a la perspectiva temporal y la regulación del peso, las investigaciones han concluido que la perspectiva temporal, particularmente el Futuro se asocia de forma significativa en la regulación del IMC (Sweeney \& Culcea, 2017; Belsky, Epel \& Tomiyama, 2014; Adams, 2012), a una conducta alimentaria saludable (Dassen, Houben \& Jansen, 2015; Hall et al., 2012) y a la realización de actividad física (Sweeney \& Culcea, 2017; Gulley, 2013; Hall et al., 2012). Por el contrario, el presente hedonista se ha asociado significativamente a la no adherencia a conductas de salud en estas esferas (Alfonsson et al., 2017). En definitiva, la perspectiva temporal hedonista puede ser un factor de riesgo para la participación en comportamientos gratificantes como comer alimentos altamente calóricos y la evitación de conductas con baja gratificación inmediata, como el ejercicio físico (Alfonsson et al., 2017).

En suma, por la evidencia revisada es necesario estudiar la interacción de las variables psicológicas: "Perspectiva Temporal", "Locus de Control" y "Autoeficacia" en pacientes sometidos a cirugía bariátrica, específicamente su capacidad para predecir la re-ganancia del peso desde el nadir.

Otro aspecto al que es necesario hacer mención, es que hasta la fecha según la búsqueda realizada en las Bases de Datos SciELO, EBSCO, WOS, ScientDirect, PsicoInfo y PudMed, la capacidad predictiva de este conjunto de variables no ha sido evaluada para la re-ganancia del peso en pacientes bariátricos.

Objetivo: Evaluar la capacidad predictiva de las variables psicológicas de la "perspectiva temporal", "locus de control del peso" y "autoeficacia para bajar de peso" para la re-ganancia del porcentaje de peso total perdido en pacientes bariátricos.

\section{Método}

Se realizó un estudio no experimental de tipo transversal bivariado y multivariado. El muestreo fue no probabilístico y la muestra estuvo constituida por 97 pacientes bariátricos. 


\section{Participantes}

Los criterios de inclusión corresponde a pacientes adultos, sometidos a cirugía bariátrica entre junio del 2002 y junio del 2017. Intervenidos con técnica quirúrgica de Bypass Gástrico o Gastrectomía vertical. Los criterios de exclusión corresponden a pacientes menores de 18 años y mayores de 70 , sometidos a cirugía bariátrica previo a junio 2002 o después de junio 2017, con técnica de intervención distinta a Bypass Gástrico o Gastrectomía vertical. Pacientes que presenten cirugía revisional o de conversión. Mujeres que se encontrasen en gestación o periodo de lactancia.

Variable de resultado:

Re-ganancia del \%PTP: Corresponde al porcentaje de recuperación de peso entre el nadir y el momento del estudio. Esta variable se analizó como variable numérica y como variable categórica a través de las siguientes ecuaciones (Véase cuadro 1). El cálculo de los \%PTP se obtuvo a partir del auto-reporte de los pesos de los pacientes.

Cuadro 1. Ecuaciones utilizadas para el calculo del \%PTP y la operacionalización de las variables de resultado

1. Ecuaciones para \%PTP:

•\%PTP_nadir $=[(\text { peso inicial }- \text { Peso nadir }) /(\text { peso inicial })]^{*} 100$

-\%PTP_actual $=[(\text { peso inicial }- \text { Peso actual }) /(\text { peso inicial })]^{*} 100$

- \% re-ganancia $=\%$ PTP_actual - \%PTP_nadir

2. Operacionalización de las variables de resultado:

- Variable numérica (regresión simple y múltiple):

$\%$ re-ganancia $=\%$ PTP_actual - \%PTP_nadir

- Variable categórica (regresión logística):

○ Mantención $=\%$ re-ganancia $<14,99 \%$

○ Re-ganancia $=\%$ re-ganancia $\geq 15 \%$

Variables de criterio e instrumentos de medición

Autoeficacia para bajar de peso, evaluada a través de la Escala de autoeficacia para disminuir de peso, construida en Chile por Campos \& Pérez (2007). La escala consta de 38 ítems, clasificados con escala Likert de 7 puntos. Evalúa tres sub-habilidades: Autoeficacia para realizar dieta (15 ítems). Autoeficacia para la realización de ejercicios (13 ítems). Autoeficacia para la compra de alimentos adecuados a una dieta (10 ítems).

Percepción de control del peso, evaluado por medio de la Escala Locus de control del peso, creada por Luciana Marín (2007). Es una escala autoadministrable, tipo Likert de cuatro alternativas. Tiene por objetivo evaluar la variable percepción de control del peso en pacientes con obesidad frente a la conducta de comer y de engordar. La escala original posee 60 ítems; 31 ítems evalúan locus de control externo (ítems inversos) y 29 locus de control interno. Mayores puntajes indican un locus de control interno.

Perspectiva Temporal, medida a través del Inventario de Perspectiva Temporal de Zimbardo, ZTPI (Zimbardo \& Boyd, 1999) fue creado en Estados Unidos y adaptado en Chile por Oyanadel, Buela-Casal \& Pérez-Fortis (2014). Tiene 56 ítems que se puntúan en una escala tipo Likert que va desde 1 a 5 . El valor más cercano a uno indica que el enunciado es menos representativo de la persona. Por el contrario un valor más cercano a cinco indica que el atributo la persona lo posee en mayor cantidad. Evalúa las cinco dimensiones de la teoría de la Orientación Temporal de Zimbardo: Pasado Positivo (PP), Pasado Negativo (PN), Presente Hedonista (PH), 
Presente Fatalista (PF) y Futuro (F). El instrumento no arroja un puntaje total, ya que cada dimensión se valora de manera independiente al ser consideradas teóricamente diferentes (Zimbardo \& Boyd, 2009).

\section{Procedimiento}

Se invitó a participar voluntariamente de la presente investigación a pacientes que se habían sometido a cirugía bariátrica. Los instrumentos fueron auto-aplicados. Los datos antropométricos (estatura, peso) fueron auto-reportados por los participantes.

\section{Aspectos Éticos}

Este estudio se fundamenta en la Tesis Doctoral "Variables Psicológicas y Predicción del éxito y fracaso en pacientes sometidos a cirugía bariátrica". Investigación revisada y aprobada por el Comité de Ética de la Universidad de Concepción, y el Comité de Ética Científica del Servicio de Salud de Concepción.

\section{Análisis de datos}

Se realizaron tres tipos de análisis de regresión. Primero, para evaluar la capacidad predictiva de cada una las variables psicológicas se efectuaron análisis regresión simple. Segundo, con el propósito de evaluar la capacidad predictiva del conjunto de variables psicológicas analizadas se realizó un análisis de regresión múltiple con procedimiento Stepwise. Tercero, finalmente se evaluó la capacidad de las variables predictivas para clasificar correctamente a los pacientes a través de un análisis de regresión logística.

\section{Resultados}

El promedio de años posoperatorio fue de 4,1 ( $\mathrm{DE}=3.4)$. La muestra estuvo conformada mayoritariamente por mujeres (89.7\%). Un 17\% presentó re-ganancia del peso desde el nadir. En la Tabla 1 se muestra la evolución del estado nutricional de la muestra.

Tabla 1. Evolución del estado nutricional de la muestra

\begin{tabular}{lcccccc}
\hline & \multicolumn{5}{c}{ Estado nutricional } \\
Momento & Normopeso & Sobrepeso & Obesidad I & Obesidad II & Obesidad III & Obesidad IV \\
\hline Previo & - & $2.1 \%$ & $16.5 \%$ & $27.8 \%$ & $43.3 \%$ & $10.3 \%$ \\
& & & & & & $2.1 \%$ \\
Nadir & $49.5 \%$ & $36.1 \%$ & $9.3 \%$ & $3.1 \%$ & $5.2 \%$ & - \\
Actual & $36.1 \%$ & $37.1 \%$ & $18.6 \%$ & $3.1 \%$ & - \\
\hline
\end{tabular}

En la Tabla 2 se muestran los descriptivos de tendencia central de las medidas psicológicas analizadas. 
Tabla 2. Descriptivos de las medidas de auto-reporte

\begin{tabular}{lc}
\hline Variable psicológica & M(DE) \\
\hline Perspectiva Temporal & - \\
Pasado Negativo & $2.98(0.76)$ \\
Pasado Positivo & $3.73(0.51)$ \\
Presente Fatalista & $2.72(0.68)$ \\
Presente Hedonista & $3.52(0.49)$ \\
Futuro & $3.82(0.49)$ \\
\hline Autoeficacia general para bajar de peso & $159.1(18.1)$ \\
\hline Para hacer dieta & $67.0(16.0)$ \\
Para hacer ejercicio & $50.4(19.9)$ \\
Para comprar alimentos & $50.8(8.9)$ \\
\hline Locus de control general del peso & $129.1(18.7)$ \\
\hline Para comer & $46.8(7.5)$ \\
$\quad$ Para adelgazar y engordar & $82.2(12.1)$ \\
\hline
\end{tabular}

En cuanto a los resultados de los análisis bivariados, las variables psicológicas "pasado positivo" $\left(\mathrm{R}^{2}=.021\right.$; $\mathrm{F}=5.64 ; \mathrm{p}<.05)$, "presente fatalista" $\left(\mathrm{R}^{2}=.042 ; \mathrm{F}=4.65 ; \mathrm{p}<.05\right)$, "presente hedonista" $\left(\mathrm{R}^{2}=.072 ; \mathrm{F}=7.39 ; \mathrm{p}<.05\right)$, "autoeficacia general para bajar de peso" $\left(\mathrm{R}^{2}=.12 ; \mathrm{F}=12.89 ; \mathrm{p}<.01\right)$ y sus tres sub-habilidades, y el "locus de control general del peso" ( $\left.\mathrm{R}^{2}=.180 ; \mathrm{F}=20.87 ; \mathrm{p}<.001\right)$ junto con sus dos sub-escalas, fueron significativas para explicar una proporción de la varianza del porcentaje de re-ganancia del peso desde el nadir hasta el momento del estudio (Véase Tabla 3).

Tabla 3. Regresiones simples para el porcentaje de re-ganancia desde el nadir.

\begin{tabular}{lcccccc}
\hline Variables psicológicas & $R^{2}$ & $\begin{array}{c}R^{2} \\
\text { ajust. }\end{array}$ & EE & F & B estandarizados & $\mathrm{t}$ \\
\hline Pasado negativo & .021 & .010 & .99 & 2.00 & .144 & 1.41 \\
Pasado positivo & .056 & .046 & .97 & 5.64 & $-.237^{*}$ & -2.37 \\
Presente fatalista & .042 & .037 & .96 & 4.65 & $-.216^{*}$ & -2.28 \\
Presente hedonista & .072 & .062 & .91 & 7.39 & $-.269 *$ & -2.72 \\
Futuro & .010 & .000 & .99 & 1.00 & -.102 & -1.00 \\
Autoeficacia para hacer dieta & .083 & .074 & .96 & 8.64 & $-.289 * *$ & -2.94 \\
Autoeficacia para hacer ejercicio & .107 & .097 & .95 & 11.36 & $-.327^{* *}$ & -3.37 \\
Autoeficacia para comprar alimentos saludables & .043 & .033 & .98 & 4.29 & $-.208^{*}$ & $-2,07$ \\
Autoeficacia general para bajar de peso & .120 & .110 & .94 & 12.89 & $-.346^{* *}$ & -3.95 \\
Locus de control para comer & .173 & .164 & .91 & 19.88 & $-.416^{* * *}$ & -4.45 \\
Locus de control para adelgazar - engordar & .157 & .148 & .92 & 17.74 & $-.397 * * *$ & -4.21 \\
Locus de control general del peso & .180 & .172 & .91 & 20.87 & $-.424 * * *$ & -4.56 \\
\hline
\end{tabular}

Nota. $* \mathrm{p}<.05 ; * * \mathrm{p}<.01 ; * * * \mathrm{p}<.001$ 
Con el propósito de analizar el porcentaje de varianza explicada por el conjunto de variables psicológicas y la capacidad predictiva de las mismas para la re-ganancia del peso, se realizó un análisis de regresión múltiple, con procedimiento Stepwise. Las variables psicológicas fueron ingresadas al análisis de acuerdo al grado de correlación arrojado en el análisis de regresión simple (coeficientes estandarizados B). El modelo incluyó a las variables "Locus de control general del peso", "Autoeficacia general para bajar de peso", "Presente hedonista", "Pasado Positivo" y "Presente Fatalista". El modelo fue significativo y explicó un $27.1 \%$ de la varianza del porcentaje de re-ganancia $\left(\mathrm{R}^{2}=0.271 ; \mathrm{F}=11.503 ; \mathrm{p}=.000\right)$. El resultado se presenta en la Tabla 4.

Tabla 4. Modelo de Regresión Múltiple con procedimiento Stepwise para el porcentaje de re-ganancia desde el nadir.

\begin{tabular}{lccccc}
\hline & B estandarizado & $\mathrm{t}$ & Sig. & Tolerancia & VIF \\
\hline Constante & & .000 & 1.000 & & \\
Locus de control general del peso & -.487 & -5.13 & .000 & .873 & 1.1 \\
Presente Fatalista & -.255 & -2.66 & .009 & .859 & 1.1 \\
Pasado Positivo & -.222 & -2.46 & .016 & .965 & 1.0 \\
Autoeficacia general para bajar de peso $^{\mathrm{a}}$ & -.154 & -1.56 & .122 & .800 & 1.2 \\
Presente Hedonista $^{\mathrm{a}}$ & -.078 & -.793 & .430 & .822 & 1.2 \\
\hline
\end{tabular}

Fuente: Elaboración propia

a Variables excluidas del modelo

En último lugar, se evaluó la capacidad de las variables psicológicas para clasificar correctamente a los pacientes en "mantención" y en "re-ganancia", para ello se realizó un análisis de regresión logística. El modelo compuesto por las variables "locus de control general del peso", "pasado positivo", y "presente fatalista" clasifica de forma correcta al $88.7 \%$ de los pacientes. El coeficiente de determinación indica que el $40.9 \%$ de la varianza de la variable dependiente está asociada al modelo (Véase Tabla 5).

Tabla 5. Resumen del modelo

\begin{tabular}{|ccc|}
\hline Logaritmo de la verosimilitud -2 & R cuadrado de Cox y Snell & R cuadrado de Nagelkerke \\
\hline 62.45 & .248 & .409 \\
\hline
\end{tabular}

Por otra parte, el modelo presenta una sensibilidad del 75\% y una especificidad del 90.5\% (Véase Tabla 6). El resumen del modelo se presenta en la Tabla 7.

Tabla 6. Clasificación Re-ganancia-Mantención del porcentaje del peso total perdido.

\begin{tabular}{lccc}
\hline & \multicolumn{3}{c}{ Pronosticado } \\
\hline Observado & Re-ganancia & Mantención & Porcentaje Correcto \\
Re-ganancia & 9 & 8 & 52.9 \\
Mantención & 3 & 77 & 96.3 \\
Total & 12 & 85 & 88.7 \\
\hline
\end{tabular}


Tabla 7. Regresión Logística del porcentaje de re-ganancia a base de variables psicológicas

\begin{tabular}{lcccccc}
\hline Variables independientes & B & EE & Wald & gl & Sig. & Exp(B) \\
\hline Locus de control general del peso & -1.28 & .360 & 12.73 & 1 & .000 & .277 \\
Pasado Positivo & -.896 & .332 & 7.30 & 1 & .007 & .408 \\
Presente Fatalista & -.898 & .371 & 5.85 & 1 & .016 & .407 \\
Constante & -2.145 & .399 & 28.92 & 1 & .000 & .117 \\
\hline
\end{tabular}

\section{Discusión}

El objetivo de este estudio fue evaluar la capacidad predictiva de las variables psicológicas "perspectiva temporal", "locus de control del peso" y "autoeficacia para bajar de peso" para la mantención y re-ganancia de la pérdida de peso en pacientes bariátricos.

En los análisis bivariados las sub-escalas de la perspectiva temporal "pasado positivo", "presente fatalista", "presente hedonista" y "desviación del perfil temporal balanceado" predicen la re-ganancia del peso. Así mismo las variables locus de control y autoeficacia, con todas sus sub-escalas, se presentaron como predictores de la variable de resultado numérica. Sin embargo, en los análisis multivariados subsiguientes, solo las variables "Locus de control general del peso", "Presente Fatalista" y "Pasado Positivo" se mantuvieron como variables predictivas significativas para la re-ganancia del peso. De igual modo, el modelo conformado por estas tres variables permitió clasificar correctamente al $88.7 \%$ de los pacientes en las categorías de "mantención" y "re-ganancia" del porcentaje de peso total perdido.

Estos resultados evidencian que un locus de control interno predice un menor porcentaje de re-ganancia en la presente muestra. Lo que se traduce en que un locus de control más interno es un factor protector para la re-ganancia del peso. En este caso, los pacientes que creen su estado nutricional actual "mantención de la pérdida del peso" depende principalmente de su comportamiento presentaron un menor porcentaje de re-ganancia del peso que los pacientes que creen que su estado nutricional actual depende principalmente de factores ajenos a su comportamiento.

Por otra parte, lo resultados obtenidos en las variables de la perspectiva temporal son importantes de analizar. Primero, porque esta investigación no apoya los hallazgos previos en relación a que el presente hedonista es factor de riesgo para la re-ganancia del peso cuando se evalúa un conjunto de predictores (Alfonsson et al., 2017). Segundo, muestra que un pasado positivo como perspectiva predominante tiene consecuencias negativas en términos de salud (Boyd \& Zimbardo, 2005), en este caso predice una mayor recuperación del peso. Lo anterior se explica debido a que una predominancia del pasado positivo interfiere con la capacidad para generar, y mantener, cambios en el estilo de vida que son necesarios cuando la capacidad y tolerancia gástrica van aumentando. Tercero, confirman que el presente fatalista se asocia a consecuencias negativas en salud, como es la re-ganancia del peso, la que trae efectos negativos a nivel de salud general y calidad de vida.

En suma, la cirugía permite un contexto de pérdida de peso que puede ser beneficioso en términos de salud y calidad de vida para el paciente, pero que en un porcentaje no menor de casos puede requerir de un trabajo multidisciplinario que le permita al paciente mantener este estado beneficioso de pérdida de peso, por lo que estos resultados son relevantes a nivel clínico y psicoterapéutico, al evidenciar hacia dónde colocar el foco psicoterapéutico en pacientes bariátricos que asisten a psicólogo por motivo de recuperación del peso.

Fortalezas: Este es el primer estudio que evalúa la capacidad predictiva de las variables "perspectiva temporal", "locus de control" y "autoeficacia" para la re-ganancia del peso en pacientes bariátricos. Los resultados evidencian la importancia de considerar estas variables en el trabajo psicoterapéutico con pacientes bariátricos.

Limitaciones: Las limitaciones de la presente investigación aluden a la variabilidad de los tiempos postoperatorios de los pacientes, y a que la muestra estuvo conformada principalmente por mujeres $(89.7 \%)$.

Perspectivas futuras: Para avanzar en la comprensión de las variables que se encuentran implicadas en la recuperación del peso, podría aportar la inclusión de otros constructos relacionados, como por ejemplo, regulación emocional. Otra área que puede colaborar en la comprensión del complejo proceso de la re-ganancia del peso, y que se ha planteado que teóricamente esta relacionada con la perspectiva temporal (Aström, Seif, Wiberg \& Carelli, 2018; Alfonsson et al., 2017), son las funciones ejecutivas. Por ejemplo, tanto la perspectiva 
temporal como las funciones ejecutivas se asocian a planificación, flexibilidad cognitiva y al comportamiento dirigido a objetivos.

\section{Referencias}

Adams, T., Davidson, L., Litwin, S., Kim, J., Kolotkin, R., Nanjee, N., ... \& McKinlay, R. (2017). Weight and metabolic outcomes 12 years after Gastric Bypass. The New England Journal of Medicine, 377, 1143 - 1155.

Adams, T., Davidson, L., Litwin, S., Kolotkin, R., LaMonte, M., Pendleton, R., ... \& Hunt, S. (2012). Health Benefits of gastric bypass surgery after 6 years. Journal of the American Medical Association, 308, 1122- 1131. doi: 10.1001/2012.jama.11164Jiménez et al., 2018.

Alfonsson, S., Weineland-Strandskov, S., \& Sundbom, M. (2017). Self-reported Hedonism Predicts 12-month weight loss after Rouxen-Y Gastric bypass. Obesity Surgery, 27, 2073-2078.

Amundsen, T., Strommen, M., \& Martins, C. (2017). Suboptimal Weight Loss and Weight Regain after Gastric Bypass Surgery-Postoperative Status of Energy Intake, Eating Behavior, Physical Activity, and Psychometrics. Obesity Surgery, 27, 1316-1326.

Anastasiou, C., Fappa, E., Karfopoulou, E., Gkza, A., \& Yannakoulia, M. (2015). Weight loss maintenance in relation to locus of control: The MedWeight study. Behaviour Research and Theraphy, 71, $40-44$.

Aström, E., Seif, A., Wiberg, B., \& Carelli, M. (2018). Getting "Stuck" in the Future or the Past: Relationships between Dimensions of Time Perspective, Executive Functions, and Repetitive Negative Thinking in Anxiety. Psychopathology, 51(6),362-370. doi: $10.1159 / 000494882$

Baird, H., Webb, T., Martin, J., \& Sirois, F. (2018). The relationship between a Balanced Time Perspective and self-monitoring of blood glucose among people with type 1 Diabetes. Annals of Behavioral Medicine, 53(2), 196-209.

Balch, P., \& Ross, A.W. (1975). Predicting sucess in weight reduction as a function of locus of control: a unidimensional and multidimensional approach. Journal of Consulting and Clinical Psychology, 43, 119.

Bas, M., \& Donmez, S. (2009). Self-efficacy and restrained eating in relation to weight loss among overweight men and women in Turkey. Appetite, 52,209-216.

Beck, N. N., Mehlson, M., \& Stoving, R. K. (2012). Psychological characteristics andassociations with weight outcomes two years after gastric bypass surgery: Postoperative eating disorder symptoms are associated with weight loss outcomes. Eating Behaviors, 13, 394-397.

Belsky, A.C.I., Epel, E.S., \& Tomiyama, A.J. (2014). Clues to maintaining calorie restriction? Psychosocial profiles of successful longterm restrictors. Appetite, 79, 106e112.

Boyd, J., \& Zimbardo, P. (2005). Time Perspective, health and risk taking. In A. Strathman \& J. Joireman (Eds.), Understanding behavior in the context of time ( $p$ p. 85-107). Mahwah: LEA.

Busseto, L., Dicker, D., Azran, C., Batterham, R., Farpour-Lambert, N., Fried, M., ... \& Yumuk, V. (2017). Practical Recommendations of the Obesity Management Task Force of the European Association for the Study of Obesity for the Post-Bariatric Surgery Medical Management. Obesity Facts, 10, 597-632.

Campos, Y., Argüelles, V., Vázquez, F., \& Ortiz, M. (2014). Autorregulación, autoeficacia y orientación nutricional para la disminución del exceso de peso. Revista Argentina de Clínica Psicológica, XXIII, 163 - 170.

Campos, S., \& Pérez J. (2007). Autoeficacia y conflicto decisional frente a la disminución del peso corporal en mujeres. Revista Chilena de Nutrición, 34, 213-218.

Clapp, B., Wynn, M., Martyn, C., Foster, C., O’Dell, M., \& Tyroch, A. (2018). Long term (7 or more years) outcomes of the sleeve gastrectomy: a meta- analysis. Surgery for Obesity and Related Diseases, 14, 741-747.

Cooper, T., Simmons, E., Webb, K., Burns, J., \& Kushner, R. (2015). Trends in weight regain following Roux-en-Y Gastic Bypass (RYGB) Bariatric Surgery. Obesity Surgery, 25, $1474-1481$.

Corcelles, R., Daigle, C.R., \& Schauer, PR. (2016). Management of endocrine disease: metabolic effects of bariatric surgery. European Journal of Endocrinology, 174, R19- R28.

Courcoulas, A., King, W., Belle, S., Berk, P., Flum, D., Garcia, L., ... Yanovski, S. (2018). Seven-Year Weight Trajectories and Health Outcomes in the Longitudinal Assessment of Bariatric Surgery (LABS) Study. JAMA Surgery, 153, 427-434.

Daugherty, J.R., \& Brase, G.L. (2010). Taking time to be healthy: Predicting health behaviors with delay discounting and time perspective. Personality and Individual Differences, 48, 202-207.

Dassen, F. C., Houben, K., \& Jansen, A. (2015). Time orientation and eating behavior: Unhealthy eaters consider immediate consequences, while healthy eaters focus on future health. Appetite, 91, 13e19.

Dawes, A.J., Maggard-Gibbons, M., Maher, A.R., Booth, M.J., Miake-Lye, I., Beroes, J.M., \& Shekelle, P.G. (2016). Mental Health Conditions Among Patients Seeking and Undergoing Bariatric Surgery: A Meta-analysis. Journal of the American Medical Association, $315,150-163$. 
Droit-Volet, S. (2013).Time perception, emotions and mood disorders. Journal of Physiology-Paris, 107, 255-264.

Franks, S. F., \& Kaiser, K.A. (2008). Predictive factors in bariatric surgery outcomes: What is the role of the preoperative psychological evaluation? Primary Psychiatry, 15, 74-83.

González, M., \& Lugli, Z. (2012). Control personal de la conducta y adhesion terapéutica en balón intragástrico Bioenterics. Gen, 66, 250-259.

Gulley, T. (2013). Time perspective and physical activity among central Appalachian adolescents. The Journal of School Nursing, 29, $123 \mathrm{e} 131$.

Hall, P.A., Fong, G.T., \& Cheng, A.Y. (2012). Time perspective and weight management behaviors in newly diagnosed Type 2 diabetes: a meditational analysis. Journal of Behavioral Medicine, 35, $569-580$.

Henson, J. M., Carey, M. P., \& Carey, K. B. (2006). Associations among health behaviors and time perspective in young adults: model testing with boot-strapping replication Journal of Behavioral Medicine, 29, 127-137.

Herpetz, S., Kielmann, R., \& Wolf, A.M. (2004). Do psychosocial variables predict weight loss or mental health after obesity surgery? A systematic review. Obesity Research, 12, 1554-1569.

Himpens, J., Dobbeleir, J., \& Peeters, G. (2010). Long-term results of laparoscopic sleeve gastrectomy for obesity. Annals of Surgery, 252, 319-324.

Ivezaj, V., \& Grilo, C. (2017). Accuracy of Self-Reported Height and Weight Among Sleeve Gastrectomy Patients with Disordered Eating. Obesity Surgery, 27, 267-270.

Jenkins, T., Boyce, T., Buncher, R., Zeller, M., Courcoulas, A., Evans, M., \& Inge, T. (2016). Accuracy of Self-reported wigth among adolescent and Young adults following bariatric surgery. Obesity Surgery, 27, 1529-1532.

Jiménez, L., Mendoca, F., Mendoza, F., Pimentel, M., Gestic, M., Chaim, E., \& Cazzo, E. (2018). Impact of Weight Regain on the Evolution of Non- alcoholic Fatty Liver Disease After Roux-en-Y Gastric Bypass: a 3-Year Follow-up. Obesity Surgery, 28, 3131-3135.

Keough, K. A., Zimbardo, P. G., \& Boyd, J. N. (1999). Who's smo- king, drinking, and using drugs? Time Perspective as a pre- dictor of substance use. Basic and Applied Social Psychology, 21, 149-164.

Kushner, R., \& Sorensen, K. (2015). Prevention of Weight Regain Following Bariatric Surgery. Current Obesity Report, 4, $198-206$.

Latner, J., McLeod, G., O’Brien, K., \& Johnston, L. (2013). The role of self-efficacy, coping, and lapses in weight maintenance. Eating and Weight Disorders - Studies on Anorexia, Bulimia and Obesity, 18, 359- 366.

Legenbauer, T., Petrak, F., de Zwaan, M., \& Herpertz, S. (2011). Influence of depressive and eating disorders on short- and long-term course of weight after surgical and nonsurgical weight loss treatment. Comprehensive Psychiatry, 52, 301-11.

Lemanu, D.P., Singh, P.P., Rahman, H., Hill, A.G., Babor, R., \& MacCormick, A.D. (2015). Five-year results after laparoscopic sleeve gastrectomy: a prospective study. Surgery for Obesity and Related Diseases, 11, 518-524.

Lent, M., Bailey-Davis, L., Irving, B., Wood, G., Cook, A., Hirrsch, A., ... \& Franceschelli-Hosterman, J. (2016). Bariatric surgery patients and their families: Health, Psysical activity, and social support. Obesity Surgery, 26, 2981- 2988.

Marín, L. (2007). Construcción y validación de un instrumento de evaluación de locus de control aplicado a pacientes obesos que buscan tratamiento médico convencional y tratamiento quirúrgico para bajar de peso (Memoria para optar al título de Psicólogo). Escuela de Ciencias Sociales, Facultad de Psicología, Universidad de Chile, Santiago.

Maciejewski, M., Arterburn, D., Van Scoyoc, L., Smith, V., Yancy, W., Weidenbacher, H., ... \& Olsen, M. (2016). Bariatric surgery and long-term durability of weight loss. JAMA Surgery, 151, 1046-1055.

Menéndez-González, L., \& Orts-Cortés, M. (2018). Psychosocial and behavioural factors in the regulation of weight: Self-regulation, self-efficacy and locus control. Enfermería clínica, 28(3), 154-161.

Morowati, M., Mazlomy, S., Baghiani M., \& Rouhani, N. (2009). Relationship between locus of control and adherence to diabetes regimen. Journal of Research in Health Sciences, 9, 37 - 44.

Neymotin, F., \& Nemzer, L. (2014). Locus of Control and Obesity. Frontiers in Endocrinology, 5, 159. doi: 10.3389/fendo.2014.00159.

Nickel, F., Schmidt, L., Bruckner, T., Büchler, M.W., Müller-Stich, B., \& Fischer, L. (2017). Influence of bariatric surgery on quality of life, body image, and general self-efficacy within 6 and 24 months - a prospective cohort study. Surgery for Obesity and Related Diseases, 13, 313-319.

Odom, J., Zalesin, K. C., Washington, T. L., Miller, W. W., Hakmeh, B., Zaremba, D. L., ... McCullough, P. A. (2010). Behavioral predictors of weight regain after bariatric surgery. Obesity Surgery, 20, 349-356.

Oyanadel, C., \& Buela-Casal, G. (2017). Percepción del tiempo y salud: La influencia del perfil temporal equilibrado (BTP) y el perfil temporal negativo (NTP) en la salud física y mental. Revista Argentina de Clínica Psicológica, 26, 296-306.

Oyanadel, C., Buela-Casal, G. \& Pérez-Fortis, A. (2014). Propiedades psicométricas del Invesntario de Orientación Temporal de Zimbardo en una muestra chilena. Terapia Psicológica, 32, 47 - 55.

Peterhänsel, C., Linde, K., Wagner, B., Dietrich, A., \& Kersting, A. (2017). Subtypes of Personality and 'Locus of Control' in Bariatric 
Patients and their Effect on Weight Loss, Eating Disorder and Depressive Symptoms, and Quality of Life. European Eating Disorders Review, 25, 397-405.

Quiñones, A., Ugarte, C., Chávez, C., \& Mañalich J. (2018). Variables psicológicas asociadas a adherencia, cronicidad y complicaciones en pacientes con diabetes mellitus II. Revista Médica de Chile, 146, 1151 - 1158.

Quiñones, A., Ceric, F., Ugarte, C., \& Pascale, A. (2017). Psychotherapy and psychological time: a case study. Rivista di Psichiatria, 52,109-116.

Quiñones, A., Ceric, F., \& Ugarte, C. (2015). Flujos de información en zonas de tiempo subjetivo: estudio de un proceso psicoterapéutico exitoso. Revista Argentina de Clínica Psicológica, XXIV, 255-266.

Rotter, J. (1966). Generalized expectancies for internal versus external control of reinforcement. Psychological Monographs: General and Applied, 80, 1-28.

Ryu, V., Kook S., Lee S.J., Ha, K., \& Cho, H.S. (2014). Effects of emotional stimuli on time perception in manic and euthymic patients with bipolar disorder. Progress in neuro-psychopharmacology \& biological psychiatry, 39, 1878-4216.

Sabench, F., Domíngez-Adame, E., Ibarzabal, A., Socas, M., Valentí, V., García, A., ... Sánchez, R. (2017). Criterios de calidad en cirugía bariátrica: revisión de conjunto y recomendaciones de la Asociación Española de Cirujanos y de la Sociedad Española de Cirugía de la Obesidad. Cirugía Española, 95, 4-16.

Shukla, A., He, D., Saunders, K., Andrew, C.. \& Aronne, L. (2018): Current concepts in management of weight regain following bariatric surgery. Expert Review of Endocrinology \& Metabolism, 13, 67-76. doi: 10.1080/17446651.2018.1447922

Schauer, P.R., Bhatt, D.L., Kirwan, J.P., Wolski, K., Aminian, A., Brethauer, S.A., ... \& STAMPEDE Investigators. (2017). Bairatric surgery versus intensive medical therapy for Diabetes - 5 year outcomes. The New England Journal of Medicine, 376, 641- 651.

Sweeney, A., \& Culcea, I. (2017).Does a future-oriented temporal perspective relate to body mass index, eating, and exercise? A meta-analysis. Appetite, 112, 272-285.

Ugarte, C., Quiñones, A., Bustos, C., \& Vicente, B. (2020, en prensa) Porcentaje de peso perdido y su recuperación en pacientes bariátricos: un análisis desde la perspectiva temporal. Revista Chilena de Nutrición, 47(1).

Ugarte, C., Quiñones, A., \& Vicente, B. (2019, en prensa). Recuperación de peso perdido en pacientes que han tenido cirugía bariátrica: Una mirada psicológica. Revista Médica de Chile, 147, 1367-1373.

Van de Laar, A., van Rijswijk, A., Kakas, H., \& Bruin, S. (2018). Sensitivity and Specificity of 50\% Excess Weight Loss (50\%EWL) and Twelve Other Bariatric Criteria for Weight Loss Success. Obesity Surgery, 28, 2297- 2304.

Van de Laar, A. (2012). Bariatric Outcomes Longitudinal Database (BOLD) suggests excess weight loss and excess BMI loss to be inappropriate outcome measures, demonstrating better alternatives. Obesity Surgery, 22, 1843-1847.

Wedin, S., Madan, A., Correll. J., Crowley, N., Malcolm, R., Byrned, K., \& Borckardt, J.J. (2014). Emotional eating, marital status and history of physical abuse predict 2-year weight loss in weight loss surgery patients. Eating Behaviors, 15, 619-624.

Weineland, S., Arvidsson, D., Kakoulidis, T., \& Dahl, J. (2011). Acceptance and commitment therapy for bariatric surgery patients, a pilot RCT. Obesisty Research \& Clinical Practice, 6, 21-30.

Wills, T.A., Sandy, J.M., \& Yaeger, A.M. (2001). Time perspective and early-onset use: a model base don stress-coping theory. Psychology of Addictive Behaviours, 15,118-25.

Zimbardo, P. G., \& Boyd, J.N. (1999). Putting time in perspective: A valid, reliable individual-differences metric. Journal of Personality and Social Psychology,77, 1271-1288.

Zimbardo, P., \& Boyd, J. (2009). La paradoja del tiempo. Barcelona: Paidós. 
